\title{
Strong Logarithmic Sobolev Inequalities for Log-Subharmonic Functions
}

\author{
Piotr Graczyk* \\ Université d'Angers, 2 Boulevard Lavoisier \\ 49045 Angers Cedex 01, France \\ Piotr.Graczyk@univ-angers.fr \\ Todd Kemp ${ }^{\dagger}$ \\ Department of Mathematics, University of California San Diego \\ 9500 Gilman Drive, La Jolla, CA 92093-0112 USA \\ tkemp@math.ucsd.edu \\ Jean-Jacques Loeb \\ Université d'Angers, 2 Boulevard Lavoisier \\ 49045 Angers Cedex 01, France \\ Jean-Jacques. Loeb@univ-angers. fr \\ January 13, 2018
}

\begin{abstract}
We prove an intrinsic equivalence between strong hypercontractivity (SHC) and a strong logarithmic Sobolev inequality (SLSI) for the cone of logarithmically subharmonic (LSH) functions. We introduce a new large class of measures, Euclidean regular and exponential type, in addition to all compactly-supported measures, for which this equivalence holds. We prove a Sobolev density theorem through LSH functions, and use it to prove the equivalence of (sHC) and (SLSI) for such log-subharmonic functions.
\end{abstract}

\section{Contents}

1 Introduction 2

1.1 Main Results . . . . . . . . . . . . . . . . . . . . . . . . 3

1.2 Alternative Formulation of $\mathrm{sHC} \ldots \ldots \ldots \ldots \ldots \ldots$

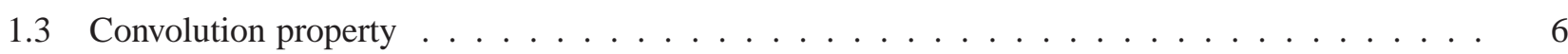

1.4 Compactly Supported Measures . . . . . . . . . . . . . . . . . 7

2 Density results through LSH functions $\quad 8$

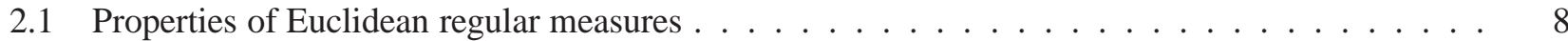

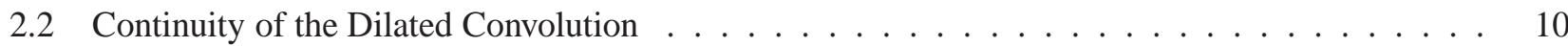

2.3 The Proof of Theorem $1.12 \ldots \ldots \ldots \ldots \ldots \ldots \ldots$

3 The Intrinsic Equivalence of (SLSI) and (sHC) 14

*Partly supported by ANR-09-BLAN-0084-01

${ }^{\dagger}$ Partly supported by NSF Grant DMS-1001894 and NSF CAREER Award DMS-1254807 


\section{Introduction}

In this paper we study strong versions of logarithmic Sobolev inequalities (SLSI) and strong hypercontractivity (SHC) in the real spaces $\mathbb{R}^{n}$ and for logarithmically subharmonic (LSH) functions, continuing our research published in [12] and solving the conjecture on the equivalence between (SHC) and (SLSI) formulated in [12. Remark 5.11]. The main difficulty to overcome, as already noticed by Gross and Grothaus in [16], was efficient approximating of (logarithmically) subharmonic functions.

If $\mu$ is a probability measure, the entropy functional $\mathrm{Ent}_{\mu}$ relative to $\mu$, defined on all sufficiently integrable positive test functions $g$, is

$$
\operatorname{Ent}_{\mu}(g)=\int g \ln \left(\frac{g}{\|g\|_{1}}\right) d \mu
$$

where $\|g\|_{1}=\|g\|_{L^{1}(\mu)}$. (When $\|g\|_{1}=1$, so $g$ is a probability density, this gives the classical Shannon entropy.) The logarithmic Sobolev inequality is an energy-entropy functional inequality: a measure $\mu$ on $\mathbb{R}^{n}$ (or more generally on a Riemannian manifold) satisfies a log Sobolev inequality if, for some constant $c>0$ and for all sufficiently smooth positive test functions $f$,

$$
\operatorname{Ent}_{\mu}\left(f^{2}\right) \leq c \int|\nabla f|^{2} d \mu
$$

Making the substitution $g=f^{2}$ gives the equivalent form $\operatorname{Ent}_{\mu}(g) \leq \frac{c}{4} \int|\nabla g|^{2} / g d \mu$, the integral on the right defining the Fisher information of $g$ relative to $\mu$. In this form, the inequality was first discovered for the standard normal law $\mu$ on $\mathbb{R}$ by Stam in [29]. It was rediscovered and named by Gross in [14], proved for standard Gaussian measures on $\mathbb{R}^{n}$ with sharp constant $c=2$. Over the past four decades, it has become an enormously powerful tool making fundamental contributions to geometry and global analysis [2, 3, 4, 5, 6, 8, 9, 10, 21, 23, 26], statistical physics [18, 31, 32, 33], mixing times of Markov chains [7, 11, 17], concentration of measure and optimal transport [22, 24, 30], random matrix theory [1, 25, 34], and many others.

Gross discovered the log Sobolev inequality through his work in constructive quantum field theory, particularly relating to Nelson's hypercontractivity estimates [28]. In fact, Gross showed in [14] that the log Sobolev inequality (LSI) is equivalent to hypercontractivity. Later, in [19, 20], Janson discovered a stronger form of hypercontractivity that holds for holomorphic test functions.

Theorem 1.1 (Janson [19]). If $\mu$ is the standard Gaussian measure on $\mathbb{C}^{n}$, and $0<p \leq q<\infty$, then for all holomorphic functions $f \in L^{p}\left(\mathbb{C}^{n}, \mu\right),\left\|f\left(e^{-t} \cdot\right)\right\|_{q} \leq\|f\|_{p}$ for $t \geq \frac{1}{2} \ln \frac{q}{p}$; for $t<\frac{1}{2} \ln \frac{q}{p}$, the dilated function $f\left(e^{-t} \cdot\right)$ is not in $L^{q}\left(\mathbb{C}^{n}, \mu\right)$.

Remark 1.2. Nelson's hypercontractivity estimates [28] involve the semigroup $e^{-t A_{\mu}}$, where $A_{\mu}$ is the Dirichlet form operator for the measure $\mu: \int|\nabla f|^{2} d \mu=\int \bar{f} A_{\mu} f d \mu$. If $d \mu=\rho d x$ has a smooth density $\rho$, integration by parts shows that $A_{\mu}=-\Delta-(\nabla \rho / \rho) \cdot \nabla$, and so when applied to holomorphic (hence harmonic) functions, $e^{-t A_{\mu}}$ is the flow of the vector field $\nabla \rho / \rho$. For the standard Gaussian measure, this is just the coordinate vector field $x$, the infinitesimal generator of dilations $E f(x)=x \cdot \nabla f(x)$, also known as the Euler operator. The perspective of this paper, like its predecessor [12], is that the strong hypercontractivity theorem is essentially about the dilation semigroup $f \mapsto f\left(e^{-t} \cdot\right)$, independent of the underlying measure.

Janson's strong hypercontractivity differs from Nelson's hypercontractivity in two important ways: first, the time-to-contraction is smaller, $\frac{1}{2} \ln \frac{q}{p}$ as opposed to the larger Nelson time $\frac{1}{2} \ln \frac{q-1}{p-1}$, and second, the theorem applies even in the regime $0<p, q<1$ where the $L^{p}$ "norms" are badly-behaved. Nevertheless, in [15], Gross showed that Janson's theorem is also a consequence of the same log Sobolev inequality (LSI); moreover, he generalized this implication considerably to complex manifolds (equipped with sufficiently nice measures). The reverse implication, however, was not established: the proof requires (LSI to hold for non-holomorphic 
functions (in particular of the form $|f|^{p / 2}$ ). We refer the reader to [12] for an extensive list of recent literature on strong hypercontractivity in the holomorphic category, and related ideas (notably reverse hypercontractivity) in the subharmonic category.

The aim of the present paper is to prove an intrinsic equivalence of strong hypercontractivity and a log Sobolev inequality. The starting point is a generalization of Theorem 1.1 beyond the holomorphic category. A function on $\mathbb{R}^{n}$ is log-subharmonic (LSH for short) if $\ln |f|$ is subharmonic; holomorphic functions are prime examples. In [12], we proved that Theorem 1.1 holds in the larger class LSH, for the Gaussian measure and several others. We also established a weak connection to a strong log Sobolev inequality.

Definition 1.3. A measure $\mu$ on $\mathbb{R}^{n}$ satisfies a strong logarithmic Sobolev inequality if there is a constant $c>0$ so that, for non-negative $g \in \mathrm{LSH}$ sufficiently smooth and integrable,

$$
\operatorname{Ent}_{\mu}(g) \leq \frac{c}{2} \int \operatorname{Egd\mu }
$$

Inequality (SLSI) could be written equivalently in the form $\operatorname{Ent}_{\mu}\left(f^{2}\right) \leq c \int f E f d \mu$; we will use it in $L^{1}$ form throughout. In [12], we showed the strong log Sobolev inequality holds for the standard Gaussian measure on $\mathbb{R}^{n}$, with constant $c=1$ (half the constant from (LSI), and conjectured that (SLSI) is equivalent in greater generality to the following form of Janson's strong hypercontractivity.

Definition 1.4. A measure $\mu$ on $\mathbb{R}^{n}$ satisfies the property of strong hypercontractivity if there is a constant $c>0$ so that, for $0<p \leq q<\infty$ and for every $f \in L^{p}(\mu) \cap \mathrm{LSH}$, we have

$$
\|f(r \cdot)\|_{L^{q}(\mu)} \leq\|f\|_{L^{p}(\mu)} \quad \text { if } \quad 0<r \leq(p / q)^{c / 2} .
$$

Remark 1.5. The statement in Definition 1.4 is given in multiplicative notation rather than additive, with $r=e^{-t}$ scaling the variable. It would appear more convenient to use the constant $c$ instead of $\frac{c}{2}$ in (SLSI) and (SHC). We choose to normalize with $\frac{c}{2}$ for historical reasons: Gross's equivalence of the log Sobolev inequality and Nelson's hypercontractivity equates $c$ in (LSI) to $\frac{c}{2}$ scaling the time to contraction.

Notation 1.6. For a function $f$ on $\mathbb{R}^{n}$ and $r \in[0,1], f_{r}$ denotes the function $f_{r}(x)=f(r x)$.

\subsection{Main Results}

In [12], we showed that (SHC) implies (SLSI) in the special case that the measure $\mu$ is compactly supported. Our first result is the converse.

Theorem 1.7. Let $\mu$ be a compactly supported measure on $\mathbb{R}^{n}$. Suppose that $\mu$ satisfies (SLSI) for all sufficiently smooth functions $g \in \mathrm{LSH}\left(\mathbb{R}^{n}\right)$. Then $\mu$ satisfies $(\underline{S H C})$ for all functions $f \in \operatorname{LSH}\left(\mathbb{R}^{n}\right)$.

Remark 1.8. We emphasize here that the domains in the equivalence consist of log-subharmonic functions a priori defined on all of $\mathbb{R}^{n}$, not just on the support of $\mu$. Indeed, the dilation semigroup is not well-defined if this is not satisfied. In fact, it is not hard to see that this result extends to log-subharmonic functions defined on any star-shaped open region containing the support of $\mu$.

Theorem 1.7 and its converse have non-trivial applications: for example, Proposition [12, Proposition 4.2] implies that (SLSI holds true for any compactly supported symmetric measure on $\mathbb{R}$, with constant $c \leq 2$. Nevertheless, it excludes the standard players in log Sobolev inequalities, most notably Gaussian measures. In [12. Theorem 5.8], we proved directly that (SLSI) holds true for the standard Gaussian measure on $\mathbb{R}^{n}$, with best constant $c=1$. This was proved directly from ( $[$ LSI $)$, and relied heavily on the precise form of the Gaussian 
measure; a direct connection to strong hypercontractivity (also proved for the Gaussian measure in [12, Theorem 3.2]) was not provided. That connection, for a wide class of measures, is the present goal.

The technicalities involved in establishing the equivalence of (SLSI) and (SHC) are challenging because of the rigidity of the class LSH: standard cut-off approximations needed to use integrability arguments in the proof are unavailable for subharmonic functions. To amend this, we use a fundamentally different approximation technique: the dilated convolution introduced in [13, 16], and developed in Section 2.2] below. In [13], the authors provided a local condition on the density of $\mu$ under which this operation is bounded on $L^{p}(\mu)$ (amounting to a bound on the Jacobian derivative of the translation and dilation). Here we present alternative conditions, which require little in terms of the local behaviour of the measure (they are essentially growth conditions near infinity) and achieve the same effect.

Definition 1.9. Let $p>0$ and let $\mu$ be a positive measure on $\mathbb{R}^{n}$ with density $\rho$. Say that $\mu$ (or $\rho$ ) is Euclidean exponential type $p$ if $\rho(x)>0$ for all $x$ and if the following two conditions hold:

$$
\begin{gathered}
\sup _{x} \sup _{|y| \leq s}|x|^{p} \frac{\rho(a x+y)}{\rho(x)}<\infty \text { for any } a>1, s \geq 0 \\
\sup _{x} \sup _{1<a<1+\epsilon} \frac{\rho(a x)}{\rho(x)}<\infty \text { for some } \epsilon>0 .
\end{gathered}
$$

If $\mu$ is Euclidean exponential type 0, we say it is Euclidean regular.

The terminology derives from the fact that conditions 1.1 and 1.2 insist that the Euclidean group acts on $\rho$ in a controlled manner; exponential type refers to the growth condition involving $|x|^{p}$ (indeed, for $p>0$ the measure must have tails that decay faster than any polynomial to be Euclidean exponential type $p$ ). For any probability measure $\mu$ with strictly positive density $\rho$, denote for $a \geq 1$ and $p, s \geq 0$

$$
C_{\mu}^{p}(a, s)=C_{\rho}^{p}(a, s) \equiv \sup _{x} \sup _{|y| \leq s}|x|^{p} \frac{\rho(a x+y)}{\rho(x)} .
$$

Then the condition that $\mu$ is Euclidean exponential type $p$ is precisely that $C_{\mu}^{p}(a, s)<\infty$ for each $a>1$ and $s \geq 0$, and $C_{\mu}^{0}(a, 0)$ is uniformly bounded for $a$ close to 1 . It is clear from the definition that $C_{\mu}^{p}(a, s)$ is an increasing function of $s$. Moreover, if $\mu$ is Euclidean exponential type $q$ then it is Euclidean exponential type $p$ for any $p<q$. For convenience, we will often write $C_{\mu}$ for $C_{\mu}^{0}$.

Example 1.10. On $\mathbb{R}$, the densities $\left(1+x^{2}\right)^{-\alpha}$ for $\alpha>\frac{1}{2}$ are Euclidean regular. On $\mathbb{R}^{n}$ the densities $e^{-c|x|^{a}}$ with $a, c>0$ are Euclidean exponential type $p$ for all $p>0$.

More examples and properties that prove the Euclidean regular measures form a rich class are given in Section 2.1. The purpose of introducing this class at present is its utility in proving a density theorem for an appropriate class of Sobolev-type spaces.

Definition 1.11. Let $\mu$ be a measure on $\mathbb{R}^{n}$, and let $p>0$. Define the Sobolev space $L_{E}^{p}(\mu)$ to consist of those weakly-differentiable functions $f \in L^{p}(\mu)$ for which $E f \in L^{p}(\mu)$. To be clear: $E f(x)=\sum_{j=1}^{n} x_{j} u_{j}(x)$, where $u_{j}$ is the function (posited to exist) satisfying

$$
-\int \partial_{j} \varphi f d x=\int \varphi u_{j} d x
$$

for any $\varphi \in C_{c}^{\infty}\left(\mathbb{R}^{n}\right)$, where $d x$ denotes Lebesgue measure. 
Standard techniques, involving approximation by $C_{c}^{\infty}$ functions, show that $L_{E}^{p}$ is dense in $L^{p}$ for reasonable measures. However, our goals here involve approximation of log-subharmonic functions, and the usual cut-off approximations fail to preserve subharmonicity. An alternative approach is to use a convolution approximate identity procedure, as is readily available for Lebesgue measure. The problem is that, for a given bump function $\varphi$, the operation $f \mapsto f * \varphi$ is typically unbounded on $L^{p}(\mu)$ when $\mu$ is not Lebesgue measure. Indeed, for $L^{p}$ of Gaussian measure, even the translation $f \mapsto f(\cdot+y)$ is unbounded. The problem is that the convolution can shift mass in from near infinity. One might hope to dilate this extra mass back out near infinity, to preserve $p$-integrability; thus the dilated convolution $f \mapsto(f * \varphi)_{r}$. Section 2.2 shows that this operation behaves well in $L^{p}$ spaces of Euclidean regular measures; it also preserves the cone LSH.

The main technical theorem of this paper is the following Sobolev density theorem.

Theorem 1.12. Let $p \in[0, \infty)$, and let $\mu$ be a Euclidean exponential type p probability measure on $\mathbb{R}^{n}$. Then the cone $\mathrm{LSH} \cap L_{E}^{p}(\mu)$ is dense in the cone $\mathrm{LSH} \cap L^{p}(\mu)$.

Using Theorem 1.12, we will prove the equivalence of (SLSI) and (sHC), the latter in a nominally weaker form.

Definition 1.13. Let $\mu$ be a probability measure on $\mathbb{R}^{n}$, and let $0<p<q<\infty$. Denote by $\operatorname{LSH}^{p<q}(\mu)$ the closure of $L^{q}(\mu) \cap \mathrm{LSH}$ in $L^{p}(\mu) \cap \mathrm{LSH}$.

For any probability measure, there is a common dense subspace $\left(L^{\infty}\right)$ for all the full $L^{q}$-spaces, $q>0$, and so the closure of $L^{q}$ in $L^{p}$ is all of $L^{p}$ for $p<q$; the proof uses cut-offs that do not respect subharmonicity, and indeed, there are no non-constant bounded subharmonic functions. In [15], Gross showed that, under certain conditions on a measure $\mu$ on a complex manifold (in terms of its Dirichlet form operator $d^{*} d$ ), in the presence of a full log Sobolev inequality (LSI), there is a common dense subspace for all holomorphic $L^{q}$ spaces of $\mu$. In the present context of logarithmically-subharmonic functions, no such technology is known, and we will content ourselves with the spaces $\mathrm{LSH}^{p<q}$. We will consider the nature of the spaces in a future publication.

This brings us to our main theorem: the equivalence of (SLSI) and (SHC) for logarithmically subharmonic functions.

Theorem 1.14. Let $\mu$ be an $O(n)$-invariant probability measure on $\mathbb{R}^{n}$.

(1) If $\mu$ is Euclidean exponential type $p$ for all $p>0$, and if $\mu$ satisfies the strong log Sobolev inequality (SLSI), then $\mu$ satisfies strong hypercontractivity (SHC) in the spaces $\mathrm{LSH}^{p<q}(\mu)$ : for $0<p \leq q<\infty$ and $f \in \mathrm{LSH}^{p<q},\left\|f_{r}\right\|_{q} \leq\|f\|_{p}$ for $0<r \leq(p / q)^{c / 2}$.

(2) If $\mu$ is Euclidean exponential type $p$ for some $p>1$, and if $\mu$ satisfies (SHC) in the above sense, then $\mu$ satisfies the strong log Sobolev inequality (SLSI):

$$
\operatorname{Ent}_{\mu}(g) \leq \frac{c}{2} \int \operatorname{Eg} d \mu
$$

for all $g \in \mathrm{LSH} \cap L_{E}^{1}(\mu) \cap C^{1}\left(\mathbb{R}^{n}\right)$.

Remark 1.15. The global assumption of rotational-invariance in Theorem 1.14 is actually quite natural in this situation. The functional $g \mapsto \int E g d \mu$ on the right-hand-side of our strong log Sobolev inequality is not generally positive, since the operator $E$ is not generally self-adjoint in $L^{2}(\mu)$; however, when $\mu$ is rotationally-invariant, this functional is positive on the cone LSH, as pointed out in [12, Proposition 5.1].

We emphasize that Theorem 1.14 is intrinsic. While the two directions of the theorem require slightly different assumptions on the applicable measures, the implications between (SLSI and (sHC) both stay within the cone LSH of log-subharmonic functions. This is the main benefit of extending Janson's strong hypercontractivity theorem from holomorphic functions to this larger class, and restricting the log-Sobolev inequality to it: here, the two are precisely equivalent. 


\subsection{Alternative Formulation of sHC}

The following equivalent characterization of strong hypercontractivity will be useful in what follows.

Proposition 1.16. Fix $c>0$ and let $q(r)$ denote the function $q(r)=r^{-2 / c}$. A measure $\mu$ satisfies strong hypercontractivity (SHC) if and only if for each function $f \in L^{1}(\mu) \cap \mathrm{LSH}$,

$$
\left\|f_{r}\right\|_{q(r)} \leq\|f\|_{1} \text { and }\left\|f_{r}\right\|_{1} \leq\|f\|_{1}, \quad \text { for } r \in(0,1] .
$$

Remark 1.17. Similarly, the form of strong hypercontractivity given in Theorem 1.14 is equivalent to the same inequalities above holding for all $f$ in the nominally smaller space $\mathrm{LSH}^{1<q(r)}$.

For the proof, it is useful to note that the class LSH is closed under $f \mapsto f^{p}$ for any $p>0$.

Proof. First, suppose (SHC) holds with constant $c$. The case $p=q=1$ yields $\left\|f_{r}\right\|_{1} \leq\|f\|_{1}$ for $0<r \leq$ $(p / q)^{2 / c}=1$. More generally, by (sHC), $\left\|f_{r}\right\|_{q} \leq\|f\|_{1}$ whenever $0<r \leq(1 / q)^{c / 2}$; i.e. whenever $q \geq r^{-c / 2}=$ $q(r)$. In particular, it follows that $\left\|f_{r}\right\|_{q(r)} \leq\|f\|_{1}$ as claimed.

Conversely, suppose the above conditions hold true. Fix $q \geq p>0$ and let $f \in L^{p}(\mu) \cap \mathrm{LSH}$. Then $f^{p} \in$ $L^{1}(\mu) \cap \mathrm{LSH}$, and so by assumption we have $\left\|\left(f^{p}\right)_{r}\right\|_{q(r)} \leq\left\|f^{p}\right\|_{1}$ for $0<r \leq 1$. Since $\left(f^{p}\right)_{r}=\left(f_{r}\right)^{p}$, it follows immediately that $\left\|f_{r}\right\|_{p \cdot q(r)}^{p} \leq\|f\|_{p}^{p}$. Setting $q=p \cdot q(r)$ and solving for $r$, we have $r=r(p, q) \equiv(p / q)^{c / 2}$, and so we have proved the equality case of (sHC). Finally, suppose that $r^{\prime} \leq r(p, q)=(p / q)^{c / 2}$; then there is $s \in$ $(0,1]$ so that $r^{\prime}=s \cdot r(p, q)$. Dilations form a multiplicative semigroup, so $f_{r^{\prime}}=\left(f_{r(p, q)}\right)_{s}$. We have just proved

that $f_{r(p, q)} \in L^{q}$, and hence $\left(f_{r(p, q)}\right)^{q}$ is in $L^{1}(\mu)$. Therefore, by assumption, $\left\|\left[\left(f_{r(p, q)}\right)^{q}\right]_{s}\right\|_{1} \leq\left\|\left(f_{r(p, q)}\right)^{q}\right\|_{1}$; unwinding this yields

$$
\left\|f_{r^{\prime}}\right\|_{q}^{q}=\left\|\left(f_{r(p, q)}\right)_{s}\right\|_{q}^{1}=\left\|\left[\left(f_{r(p, q)}\right)_{s}\right]^{q}\right\|_{1}=\left\|\left[\left(f_{r(p, q)}\right)^{q}\right]_{s}\right\|_{1} \leq\left\|\left(f_{r(p, q)}\right)^{q}\right\|_{1}=\left\|f_{r(p, q)}\right\|_{q}^{q} \leq\|f\|_{p}^{q}
$$

by the equality case, thus proving (sHC).

Remark 1.18. In fact, (SHC) implies the putatively stronger statement that $r \mapsto\left\|f_{r}\right\|_{q(r)}$ is non-decreasing on $[0,1]$; however, the weaker form presented above is generally easier to work with.

\subsection{Convolution property}

We will use the convolution operation to prove the Sobolev density theorem at the heart of this paper, as well as Theorem 1.7. We begin by showing that this operation preserves the cone LSH.

Lemma 1.19. Let $f \in \mathrm{LSH}$. Let $\varphi \geq 0$ be a $C_{c}^{\infty}$ test function. Then $f * \varphi \in \mathrm{LSH} \cap C^{\infty}$.

Proof. Since $f \in \mathrm{LSH}, f \geq 0$ and $\ln f$ is subharmonic. In particular, $\ln f$ is upper semi-continuous and locally bounded above, and so the same holds for $f$. Thus $f$ is locally bounded and measurable; thus $f * \varphi$ defines an $L_{\text {loc }}^{1} \cap C^{\infty}$ function. We must show it is LSH.

Any subharmonic function is the decreasing limit of a sequence of $C^{\infty}$ subharmonic functions, cf. [27, Appendix 1, Proposition 1.15]. Applying this to $\ln f$, there is a sequence $f_{n} \in \mathrm{LSH} \cap C^{\infty}$ such that $f_{n} \downarrow f$. Let $g_{n}=f_{n}+\frac{1}{n}$; so $g_{n}$ is strictly positive, and $g_{n} \downarrow f$. Since $\varphi$ is $\geq 0$, it follows from the Monotone Convergence Theorem that $g_{n} * \varphi \downarrow f * \varphi$ pointwise.

Now, $\left(g_{n} * \varphi\right)(x)=\int_{\mathbb{R}^{n}} g_{n}(x-\omega) \varphi(\omega) d \omega$. Since translation and positive dilation preserve the cone LSH, the function $x \mapsto g_{n}(x-\omega) \varphi(\omega)$ is continuous and LSH for each $\omega$. Moreover, the function $\omega \mapsto g_{n}(x-\omega) \varphi(\omega)$ is continuous and bounded. Finally, for $\operatorname{small}, \sup _{|t-x| \leq r} g_{n}(t-\omega) \varphi(\omega) \leq\|\varphi\|_{\infty} \sup _{|t| \leq|x|+r} g_{n}(t)$ is bounded uniformly in $\omega$. It follows from [12, Lemma 2.4] that $g_{n} * \varphi$ is LSH. (The statement of that lemma apparently 
requires the supremum to be uniform in $x$ as well, but this is an overstatement; as the proof of the lemma clearly shows, only uniformity in $\omega$ is required).

Thus, $f * \varphi$ is the decreasing limit of strictly positive LSH functions $g_{n} * \varphi$. Applying the Monotone Convergence Theorem to integrals of $\ln \left(g_{n} * \varphi\right)$ about spheres now shows that $\ln (f * \varphi)$ is subharmonic, so $f * \varphi \in \operatorname{LSH}$ as claimed.

\subsection{Compactly Supported Measures}

This section is devoted to the proof of Theorem 1.7 It follows the now-standard Gross proof of such equivalence: differentiating hypercontractivity at the critical time yields the log Sobolev inequality, and vice versa. The technical issues related to differentiating under the integral can be dealt with fairly easily in the case of a compactly supported measure; the remainder of this paper develops techniques for handling measures with non-compact support. The forward direction of the theorem, that (SHC) implies SLSI for compactly supported measures, is [12, Theorem 5.2], so we will only include the proof of the reverse direction here.

Proof of Theorem 1.7 By assumption, (SLSI holds for sufficiently smooth and integrable functions; here we interpret that precisely to mean $\operatorname{Ent}_{\mu}(g) \leq \frac{c}{2} \int E g d \mu$ for all $g \in C^{1}\left(\mathbb{R}^{n}\right)$ for which both sides are finite. Fix $f \in L^{1}(\mu) \cap \mathrm{LSH} \cap C^{1}$. Utilizing Proposition 1.16, we must consider the function $\alpha(r)=\left\|f_{r}\right\|_{q(r)}$ where $q(r)=$ $r^{-2 / c}$. Let $\beta(r)=\alpha(r)^{q(r)}=\int f(r x)^{q(r)} \mu(d x)$ and set $\beta_{x}(r)=f(r x)^{q(r)}$ so that $\beta(r)=\int \beta_{x}(r) \mu(d x)$. Then,

$$
\frac{\partial}{\partial r} \ln \beta_{x}(r)=q^{\prime}(r) \ln f(r x)+\frac{q(r)}{f(r x)} x \cdot \nabla f(r x) .
$$

Since $q^{\prime}(r)=-\frac{2}{r c} q(r)$, and since $x \cdot \nabla f(r x)=\frac{1}{r}(E f)_{r}(x)=\frac{1}{r} E\left(f_{r}\right)(x)$, we have

$$
\frac{\partial}{\partial r} \beta_{x}(r)=-\frac{2}{r c} f_{r}(x)^{q(r)} \ln f_{r}(x)^{q(r)}+\frac{1}{r} q(r) f_{r}(x)^{q(r)-1}\left(E f_{r}\right)(x) .
$$

Fix $0<\epsilon<1$. As $f$ is $C^{1}$, the function (of $x$ ) on the right-hand-side of (1.4) is uniformly bounded for $r \in(\epsilon, 1]$ and $x \in \operatorname{supp} \mu$ (due to compactness). The Dominated Convergence Theorem thus allows differentiation under the integral, and so

$$
\beta^{\prime}(r)=\int \frac{\partial}{\partial r} \beta_{x}(r) \mu(d x) .
$$

Thus, since $\alpha(r)=\beta(r)^{1 / q(r)}$ and $\beta(r)>0$, it follows that $\alpha$ is $C^{1}$ on $(\epsilon, 1]$ and the chain rule yields

$$
\alpha^{\prime}(r)=\frac{\alpha(r)}{q(r) \beta(r)} \frac{2}{r c}\left[\beta(r) \ln \beta(r)+\frac{r c}{2} \beta^{\prime}(r)\right] .
$$

From (1.4) and (1.5), the quantity in brackets is

$$
\begin{aligned}
& \int f_{r}^{q(r)} d \mu \cdot \ln \int f_{r}^{q(r)} d \mu+\frac{r c}{2} \int\left(-\frac{2}{r c} f_{r}^{q(r)} \ln f_{r}^{q(r)}+\frac{1}{r} q(r) f_{r}^{q(r)-1} E f_{r}\right) d \mu \\
= & \int f_{r}^{q(r)} d \mu \cdot \ln \int f_{r}^{q(r)} d \mu-\int f_{r}^{q(r)} \ln f_{r}^{q(r)} d \mu+q(r) \frac{c}{2} \int f_{r}^{q(r)-1} E f_{r} d \mu \\
= & -\operatorname{Ent}_{\mu}\left(f_{r}^{q(r)}\right)+\frac{c}{2} \int E\left(f_{r}^{q(r)}\right) d \mu,
\end{aligned}
$$

where the equality in the last term follows from the chain rule.

Since $f \in C^{1}$, it is bounded on the compact set $\operatorname{supp} \mu$, and so are all of its dilations $f_{r}$. Hence, both terms in (1.7) are finite, and so by the assumption of the theorem, this term is $\geq 0$. From (1.6), we therefore have 
$\alpha^{\prime}(r) \geq 0$ for all $r>\epsilon$. Since this is true for each $\epsilon>0$, it holds true for $r \in(0,1]$. This verifies the first inequality in Proposition 1.16 For the second, we use precisely the same argument to justify differentiating under the integral to find

$$
\frac{\partial}{\partial r}\left\|f_{r}\right\|_{1}=\int \frac{\partial}{\partial r} f_{r}(x) \mu(d x)=\frac{1}{r} \int E f_{r}(x) \mu(d x) \geq \frac{2}{c r} \operatorname{Ent}_{\mu}\left(f_{r}\right) \geq 0
$$

by the assumption of (SLSI). This concludes the proof for $f \in C^{1}$.

Now, if $f \in L^{1}(\mu) \cap \mathrm{LSH}$, we consider a smooth approximate identity sequence $\varphi_{k}$. The inequalities in Proposition 1.16 hold for $f * \varphi_{k}$ by the first part of the proof and Lemma 1.19 Note by simple change of variables that $\left(f * \varphi_{k}\right)_{r}=f_{r} *\left(r^{n} \varphi_{k}\right)_{r}$, and that $\left(r^{n} \varphi_{k}\right)_{r}$ is also an approximate identity sequence. The function $f_{r}$ is LSH, so it is upper semi-continuous and consequently locally bounded. Thus $f_{r} \in L^{q(r)}$ and $\left(f * \varphi_{k}\right)_{r}$ converges to $f_{r}$ in $L^{q(r)}$. This concludes the proof.

\section{Density results through LSH functions}

\subsection{Properties of Euclidean regular measures}

In this section, we show several closure properties of the class of Euclidean regular measures (of any given exponential type $p \in[0, \infty)$ ): it is closed under bounded perturbations, convex combinations, product, and convolution. Throughout, we use $\mu_{i}(i=1,2)$ to stand for such measures, and $\rho_{i}$ to stand for their densities.

Proposition 2.1. Let $\mu_{1}$ and $\mu_{2}$ be positive measures on $\mathbb{R}^{n}$, and suppose $\mu_{1}$ is Euclidean exponential type $p \in[0, \infty)$. If there are constants $C, D>0$ such that $C \mu_{1} \leq \mu_{2} \leq D \mu_{1}$, then $\mu_{2}$ is also Euclidean exponential type $p$.

Proof. The assumption is that $C \rho_{1} \leq \rho_{2} \leq D \rho_{1}$. Let $\epsilon>0$ be such that $\sup _{1<a<1+\epsilon} C_{\rho_{1}}^{0}(a, 0)<\infty$. Then for any such $a$,

$$
\frac{\rho_{2}(a x)}{\rho_{2}(x)} \leq \frac{D \rho_{1}(a x)}{C \rho_{1}(x)} \leq \frac{D}{C} C_{\rho_{1}}^{0}(a, 0)
$$

for all $x$; thus $C_{\rho_{2}}^{0}(a, 0) \leq \frac{D}{C} C_{\rho_{1}}^{0}(a, 0)$, and so $\sup _{1<a<1+\epsilon} C_{\rho_{2}}^{0}(a, 0)<\infty$. Similarly, for $x, y \in \mathbb{R}^{n}$ and $a>1$,

$$
|x|^{p} \frac{\rho_{2}(a x+y)}{\rho_{2}(x)} \leq|x|^{p} \frac{D \rho_{1}(a x+y)}{C \rho_{1}(x)} \leq \frac{D}{C} C_{\rho_{1}}^{p}(a,|y|)
$$

and so $C_{\rho_{2}}^{p}(a, s) \leq \frac{D}{C} C_{\rho_{1}}^{p}(a, s)<\infty$.

Proposition 2.2. Let $\mu_{1}$ and $\mu_{2}$ be Euclidean regular measures of exponential type $p \in[0, \infty)$. For any $t \in[0,1]$, $\mu=(1-t) \mu_{1}+t \mu_{2}$ is Euclidean exponential type $p$.

Proof. Let $\epsilon>0$ be such that $\sup _{1<a<1+\epsilon} C_{\rho_{i}}^{0}(a, 0)<\infty$ for $i=1,2$. Let $\rho$ be the density of $\mu$. Then for any $x \in \mathbb{R}^{n}$,

$$
\begin{aligned}
\rho(a x)=(1-t) \rho_{1}(a x)+t \rho_{2}(a x) & \leq(1-t) C_{\rho_{1}}^{0}(a, 0) \rho_{1}(x)+t C_{\rho_{2}}^{0}(a, 0) \rho_{2}(x) \\
& \leq \max \left\{C_{\rho_{1}}^{0}(a, 0), C_{\rho_{2}}^{0}(a, 0)\right\} \rho(x)
\end{aligned}
$$

and so $C_{\rho}^{0}(a, 0) \leq \max \left\{C_{\rho_{1}}^{0}(a, 0), C_{\rho_{2}}^{0}(a, 0)\right\}$ is uniformly bounded for $1<a<1+\epsilon$, as required. Similarly, for $x, y \in \mathbb{R}^{n}$ and $a>1$,

$$
\begin{aligned}
|x|^{p} \rho(a x+y) \leq(1-t)|x|^{p} \rho_{1}(a x+y)+t|x|^{p} \rho_{2}(a x+y) & \leq(1-t) C_{\rho_{1}}^{p}(a,|y|) \rho_{1}(x)+t C_{\rho_{2}}^{p}(a,|y|) \rho_{2}(x) \\
& \leq \max \left\{C_{\rho_{1}}^{p}(a,|y|), C_{\rho_{2}}^{p}(a,|y|)\right\} \rho(x)
\end{aligned}
$$

which shows that $C_{\rho}^{p}(a, s) \leq \max \left\{C_{\rho_{1}}^{p}(a, s), C_{\rho_{2}}^{p}(a, s)\right\}<\infty$ for $a \geq 1$ and $s \geq 0$. 
Proposition 2.3. Let $p \in[0, \infty)$, let $\mu_{1}$ be a Euclidean exponential type $p$ measure on $\mathbb{R}^{n_{1}}$, and let Let $\mu_{2}$ be a Euclidean exponential type p measure on $\mathbb{R}^{n_{2}}$. Then the product measure $\mu_{1} \otimes \mu_{2}$ is Euclidean exponential type p on $\mathbb{R}^{n_{1}+n_{2}}$.

Proof. For $i=1,2$ let $\rho_{i}$ be the density of $\mu_{i}$; then $\mu_{1} \otimes \mu_{2}$ has density $\rho_{1} \otimes \rho_{2}\left(x_{1}, x_{2}\right)=\rho_{1}\left(x_{1}\right) \rho_{2}\left(x_{2}\right)$. Fix $\epsilon>0$ so that $\sup _{1<a<1+\epsilon} C_{\rho_{i}}^{p}(0, a)<\infty$ for $i=1,2$. Then, letting $\mathbf{x}=\left(x_{1}, x_{2}\right)$,

$$
\rho_{1} \otimes \rho_{2}(a \mathbf{x})=\rho_{1}\left(a x_{1}\right) \rho_{2}\left(a x_{2}\right) \leq C_{\rho_{1}}^{0}(a, 0) \rho_{1}\left(x_{1}\right) \cdot C_{\rho_{2}}^{0}(a, 0) \rho_{2}\left(x_{2}\right)
$$

and so $C_{\rho_{1} \otimes \rho_{2}}^{0}(a, 0) \leq C_{\rho_{1}}^{0}(a, 0) \cdot C_{\rho_{2}}^{0}(a, 0)$, meaning $\sup _{1<a<1+\epsilon} C_{\rho_{1} \otimes \rho_{2}}^{0}(a, 0)<\infty$. Similarly, for fixed $\mathbf{x}, \mathbf{y} \in \mathbb{R}^{n_{1}+n_{2}}$ and $a>1$,

$$
|\mathbf{x}|^{p} \rho_{1} \otimes \rho_{2}(a \mathbf{x}+\mathbf{y})=\left(\left|x_{1}\right|+\left|x_{2}\right|\right)^{p} \rho_{1}\left(a x_{1}+y_{1}\right) \rho_{2}\left(a x_{2}+y_{2}\right) .
$$

By elementary calculus, $\left(\left|x_{1}\right|+\left|x_{2}\right|\right)^{p} \leq 2^{p-1}\left(\left|x_{1}\right|^{p}+\left|x_{2}\right|^{p}\right)$, and so we have

$$
|\mathbf{x}|^{p} \rho_{1} \otimes \rho_{2}(a \mathbf{x}+\mathbf{y}) \leq 2^{p-1}\left|x_{1}\right|^{p} \rho_{1}\left(a x_{1}+y_{1}\right) \cdot \rho_{2}\left(a x_{2}+y_{2}\right)+2^{p-1} \rho_{1}\left(a x_{1}+y_{1}\right) \cdot\left|x_{2}\right|^{p} \rho_{2}\left(a x_{2}+y_{2}\right) .
$$

For the first term, we have $\left|x_{1}\right|^{p} \rho_{1}\left(a x_{1}+y_{1}\right) \leq C_{\rho_{1}}^{p}\left(a,\left|y_{1}\right|\right) \rho_{1}\left(x_{1}\right)$ while $\rho_{2}\left(a x_{2}+y_{2}\right) \leq C_{\rho_{2}}^{0}\left(a,\left|y_{2}\right|\right)$; for the second term, we have $\rho_{1}\left(a x_{1}+y_{1}\right) \leq C_{\rho_{1}}^{0}\left(a,\left|y_{1}\right|\right) \rho_{1}\left(x_{1}\right)$ while $\left|x_{2}\right|^{p} \rho_{2}\left(a x_{2}+y_{2}\right) \leq C_{\rho_{2}}^{p}\left(a,\left|y_{2}\right|\right)$. If $|y| \leq s$ then $\left|y_{i}\right| \leq s$ for $i=1,2$. All together, this shows that

$$
C_{\rho_{1} \otimes \rho_{2}}^{p}(a, s) \leq 2^{p-1}\left[C_{\rho_{1}}^{p}(a, s) C_{\rho_{2}}^{0}(a, s)+C_{\rho_{1}}^{0}(a, s) C_{\rho_{2}}^{p}(a, s)\right]
$$

which is finite since both $\rho_{1}, \rho_{2}$ are Euclidean exponential type $p$ (and hence also Euclidean regular). This proves the proposition.

Proposition 2.4. Let $\mu_{1}$ and $\mu_{2}$ be positive measures on $\mathbb{R}^{n}$, each of Euclidean exponential type $p \in[0, \infty)$. Then $\mu_{1} * \mu_{2}$ is Euclidean exponential type $p$.

Proof. Let $\rho_{j}$ be the density of $\mu_{j}$. By assumption, for $i=1,2 C_{\rho_{i}}^{p_{i}}(a, s)<\infty$ for $a>1$ and $s \geq 0$, and there is $\epsilon>0$ such that $\sup _{1<a<1+\epsilon} C_{\rho_{i}}^{0}(a, 0)<\infty$, cf. (1.3). Then for $a \geq 1$ and $x \in \mathbb{R}^{n}$

$$
\rho_{1} * \rho_{2}(a x)=\int \rho_{1}(a x-u) \rho_{2}(u) d u=a^{n} \int \rho_{1}(a x-a v) \rho_{2}(a v) d v .
$$

By definition, $\rho_{1}(a(x-v)) \leq C_{\rho_{1}}^{0}(a, 0) \rho_{1}(x-v)$ and $\rho_{2}(a v) \leq C_{\rho_{2}}^{0}(a, 0) \rho_{2}(v)$ for all $x, v$. Thus

$$
\rho_{1} * \rho_{2}(a x) \leq a^{n} C_{\rho_{1}}^{0}(a, 0) \cdot C_{\rho_{2}}^{0}(a, 0) \int \rho_{1}(x-v) \rho_{2}(v) d v=a^{n} C_{\rho_{1}}^{0}(a, 0) \cdot C_{\rho_{2}}^{0}(a, 0) \rho_{1} * \rho_{2}(x) .
$$

It follows that $C_{\rho_{1} * \rho_{2}}^{0}(a, 0) \leq a^{n} C_{\rho_{1}}^{0}(a, 0) \cdot C_{\rho_{2}}^{0}(a, 0)$, and hence

$$
\sup _{1<a<1+\epsilon} C_{\rho_{1} * \rho_{2}}^{0}(a, 0) \leq(1+\epsilon)^{n} \sup _{1<a<1+\epsilon} C_{\rho_{1}}^{0}(a, 0) \cdot \sup _{1<a<1+\epsilon} C_{\rho_{2}}^{0}(a, 0)<\infty
$$

as required. Similarly, for $x, y \in \mathbb{R}^{n}$ and $a>1$,

$$
|x|^{p} \rho_{1} * \rho_{2}(a x+y)=|x|^{p} \int \rho_{1}(a x+y-u) \rho_{2}(u) d u=a^{n} \int|x|^{p} \rho_{1}(a(x-v)+y) \rho_{2}(a v) d v .
$$

Note (by elementary calculus) that $|x|^{p} \leq 2^{p-1}\left(|x-v|^{p}+|v|^{p}\right)$, and so

$$
|x|^{p} \rho_{1} * \rho_{2}(a x+y) \leq 2^{p-1} a^{n}\left[\int|x-v|^{p} \rho_{1}(a(x-v)+y) \rho_{2}(a v) d v+\int \rho_{1}(a(x-v)+y)|v|^{p} \rho(a v) d v\right] .
$$


In the first term, we have $|x-v|^{p} \rho_{1}(a(x-v)+y) \leq C_{\rho_{1}}^{p}(a,|y|) \rho_{1}(x-v)$ and $\rho_{2}(a v) \leq C_{\rho_{2}}^{0}(a, 0)$, and so

$$
\int|x-v|^{p} \rho_{1}(a(x-v)+y) \rho_{2}(a v) d v \leq C_{\rho_{1}}^{p}(a,|y|) \cdot C_{\rho_{2}}^{0}(a, 0) \rho_{1} * \rho_{2}(x) .
$$

In the second term, we have $\rho_{1}(a(x-v)+y) \leq C_{\rho_{1}}^{0}(a,|y|) \rho_{1}(x-v)$ and $|v|^{p} \rho(a v) \leq C_{\rho_{2}}^{p}(a, 0)$, and so

$$
\int \rho_{1}(a(x-v)+y)|v|^{p} \rho(a v) d v \leq C_{\rho_{1}}^{0}(a,|y|) \cdot C_{\rho_{2}}^{p}(a, 0) \rho_{1} * \rho_{2}(x) .
$$

All together, for any $s \geq|y|$, this gives

$$
C_{\rho_{1} * \rho_{2}}^{p}(a, s) \leq 2^{p-1} a^{n}\left[C_{\rho_{1}}^{p}(a, s) \cdot C_{\rho_{2}}^{0}(a, 0)+C_{\rho_{1}}^{0}(a, s) \cdot C_{\rho_{2}}^{p}(a, 0)\right]
$$

which is finite since both $\rho_{1}$ and $\rho_{2}$ are Euclidean exponential type $p$ (and thus also Euclidean regular). Equations (2.1) and (2.2) prove the proposition.

\subsection{Continuity of the Dilated Convolution}

One easy consequence of Definition 1.9 is that the operation $f \mapsto f_{r}$ is bounded on $L^{p}$.

Lemma 2.5. Let $\mu$ be a Euclidean regular probability measure, let $p>0$, and let $r \in(0,1)$. Then

$$
\left\|f_{r}\right\|_{L^{p}(\mu)} \leq r^{-n / p} C_{\mu}\left(\frac{1}{r}, 0\right)^{1 / p}\|f\|_{L^{p}(\mu)} .
$$

Proof. We simply change variables $u=r x$ and use Definition 1.9 .

$$
\int\left|f_{r}(x)\right|^{p} \mu(d x)=\int|f(r x)|^{p} \rho(x) d x=r^{-n} \int|f(u)|^{p} \rho(x / r) d x \leq r^{-n} C_{\mu}\left(\frac{1}{r}, 0\right) \int|f(u)|^{p} \rho(x) d x .
$$

Remark 2.6. By condition (1.2) of Definition 1.9, the constant in Lemma2.5 is uniformly bounded for $r \in(\epsilon, 1]$ for any $\epsilon>0$; that is, there is a uniform (independent of $r$ ) constant $C_{\epsilon}$ so that, for $r \in(\epsilon, 1],\left\|f_{r}\right\|_{L^{p}(\mu)} \leq$ $C_{\epsilon}\|f\|_{L^{p}(\mu)}$.

The next proposition shows that, under the assumptions of Definition 1.9 the dilated convolution operation is indeed bounded on $L^{p}$. As usual, the conjugate exponent $p^{\prime}$ to $p \in[1, \infty)$ is defined by $\frac{1}{p}+\frac{1}{p^{\prime}}=1$.

Proposition 2.7. Let $\mu$ be a Euclidean regular probability measure on $\mathbb{R}^{n}$. Let $p \in[1, \infty)$, and let $\varphi \in C_{c}^{\infty}$ be a test function. Then the dilated convolution operation $f \mapsto(f * \varphi)_{r}$ is bounded on $L^{p}(\mu)$ for each $r \in(0,1)$. Precisely, if $K=\operatorname{supp} \varphi$ and $s=\sup \{|w| ; w \in K\}$, then

$$
\left\|(f * \varphi)_{r}\right\|_{L^{p}(\mu)} \leq r^{-n / p} C_{\mu}\left(\frac{1}{r}, \frac{s}{r}\right)^{1 / p} \operatorname{Vol}(K)^{1 / p}\|\varphi\|_{L^{p^{\prime}}(K)}\|f\|_{L^{p}(\mu)},
$$

where $C_{\mu}$ is the constant defined in (1.3).

Proof. Denote by $K$ the support of $\varphi$. By definition,

$$
\left\|(f * \varphi)_{r}\right\|_{L^{p}(\mu)}^{p}=\int_{\mathbb{R}^{n}}\left|\int_{K} f(r x-y) \varphi(y) d y\right|^{p} \rho(x) d x .
$$


We immediately estimate the internal integral using Hölder's inequality:

$$
\left|\int_{K} f(r x-y) \varphi(y) d y\right|^{p} \leq \int_{K}|f(r x-y)|^{p} d y \cdot\|\varphi\|_{L^{p^{\prime}(K)}}^{p}
$$

which is finite since the first integral is the $p$-th power of the $L^{p}$-norm of $f$ restricted to the compact set $r x-K$. Hence,

$$
\left\|(f * \varphi)_{r}\right\|_{L^{p}(\mu)}^{p} \leq\|\varphi\|_{L^{p^{\prime}(K)}}^{p} \int_{\mathbb{R}^{n}} \int_{K}|f(r x-y)|^{p} d y \rho(x) d x .
$$

We apply Fubini's theorem to the double integral, which is therefore equal to

$$
\int_{K} \int_{\mathbb{R}^{n}}|f(r x-y)|^{p} \rho(x) d x d y=\int_{K} r^{-n} \int_{\mathbb{R}^{n}}|f(u)|^{p} \rho\left(\frac{u+y}{r}\right) d u d y
$$

where we have made the change of variables $u=r x-y$ in the internal integral. By assumption, $\rho$ is Euclidean regular, and so we have

$$
\rho\left(\frac{1}{r} u+\frac{1}{r} y\right) \leq C_{\mu}\left(\frac{1}{r}, \frac{s}{r}\right) \rho(u), \quad y \in K .
$$

where $s=\sup \{|w| ; w \in K\}$. Substituting (2.5) into (2.4), we see that (2.3) yields

$$
\left\|(f * \varphi)_{r}\right\|_{L^{p}(\mu)}^{p} \leq r^{-n} C_{\mu}\left(\frac{1}{r}, \frac{s}{r}\right) \operatorname{Vol}(K)\|\varphi\|_{L^{p^{\prime}(K)}}^{p} \int|f(u)|^{p} \rho(u) d u .
$$

This completes the proof.

Remark 2.8. The explicit constant in Proposition 2.7 appears to depend strongly on the support set of $\varphi$, but it does not. Indeed, it is easy to check that the standard rescaling of a test function, $\varphi^{s}(x)=s^{-n} \varphi(x / s)$, which preserves total mass, also preserves the $\varphi$-dependent quantity above; to be precise, $\operatorname{Vol}\left(\operatorname{supp} \varphi^{s}\right)\left\|\varphi^{s}\right\|_{L^{p^{\prime}}\left(\mathbb{R}^{n}\right)}^{p}$ does not vary with $s$. In addition, the constant $C_{\mu}(1 / r, s / r)$ is well-behaved as $s$ shrinks (indeed, it only decreases). It is for this reason that the proposition allows us to use the dilated convolution operation with an approximate identity sequence in what follows.

The use of Proposition 2.7 is that it allows us to approximate an $L^{p}$ function by smoother $L^{p}$ functions, along a path through LSH functions. To prove this, we first require the following continuity lemma.

Lemma 2.9. Let $\mu$ be a Euclidean regular probability measure, and let $r \in(0,1)$. Then for any $f \in L^{p}(\mu)$, the map $T_{f}: \mathbb{R}^{n} \rightarrow L^{p}(\mu)$ given by $\left[T_{f}(y)\right](x)=f_{r}(x-y)$ is continuous.

Proof. First note that, by the change of variables $u=r x-r y$,

$$
\left\|T_{f}(y)\right\|_{L^{p}(\mu)}^{p}=\int|f(r x-r y)|^{p} \rho(x) d x=r^{-n} \int|f(u)|^{p} \rho\left(\frac{1}{r} u+y\right) d u,
$$

and the latter is bounded above by $r^{-n} C_{\mu}\left(\frac{1}{r},|y|\right)\|f\|_{L^{p}(\mu)}^{p}$, showing that the range of $T_{f}$ is truly in $L^{p}(\mu)$ for $y \in \mathbb{R}^{n}$. Now, fix $\epsilon>0$ and let $\psi \in C_{c}\left(\mathbb{R}^{n}\right)$ be such that $\|f-\psi\|_{L^{p}(\mu)}<\epsilon$. Let $\left(y_{k}\right)_{k=1}^{\infty}$ be a sequence in $\mathbb{R}^{n}$ with limit $y_{0}$. Then

$$
\left\|T_{f}\left(y_{k}\right)-T_{f}\left(y_{0}\right)\right\|_{L^{p}(\mu)} \leq\left\|T_{f}\left(y_{k}\right)-T_{\psi}\left(y_{k}\right)\right\|_{L^{p}(\mu)}+\left\|T_{\psi}\left(y_{k}\right)-T_{\psi}\left(y_{0}\right)\right\|_{L^{p}(\mu)}+\left\|T_{\psi}\left(y_{0}\right)-T_{f}\left(y_{0}\right)\right\|_{L^{p}(\mu)} .
$$

The first and last terms are simply $T_{\psi-f}\left(y_{k}\right)$ (with $k=0$ for the last term), and so we have just proved that

$$
\left\|T_{\psi-f}\left(y_{k}\right)\right\|_{L^{p}(\mu)} \leq r^{-n / p} C_{\mu}\left(\frac{1}{r},\left|y_{k}\right|\right)^{1 / p}\|\psi-f\|_{L^{p}(\mu)}<r^{-n / p} C_{\mu}\left(\frac{1}{r},\left|y_{k}\right|\right)^{1 / p} \epsilon .
$$


Moreover, there is a constant $s$ so that $\left|y_{k}\right| \leq s$ for all $k$, and since $C_{\mu}(a, s)$ is an increasing function of $s$, it follows that

$$
\left\|T_{f}\left(y_{k}\right)-T_{f}\left(y_{0}\right)\right\|_{L^{p}(\mu)} \leq\left\|T_{\psi}\left(y_{k}\right)-T_{\psi}\left(y_{0}\right)\right\|_{L^{p}(\mu)}+2 r^{-n / p} C_{\mu}\left(\frac{1}{r}, s\right)^{1 / p} \epsilon .
$$

For each $x,\left(T_{\psi}\left(y_{k}\right)(x)-T_{\psi}\left(y_{0}\right)(x)=\psi\left(r x-r y_{k}\right)-\psi\left(r x-r y_{0}\right)\right.$ converges to 0 since $r y_{k} \rightarrow r y_{0}$ and $\psi$ is continuous. In addition, $\psi_{r}$ is compactly supported and continuous, so it is uniformly bounded. Since $\mu$ is a probability measure, it now follows that $\left\|T_{\psi}\left(y_{k}\right)-T_{\psi}\left(y_{0}\right)\right\|_{L^{p}(\mu)} \rightarrow 0$ as $y_{k} \rightarrow y_{0}$, and the lemma follows by letting $\epsilon \downarrow 0$.

Corollary 2.10. Let $\mu$ be a Euclidean regular probability measure, and let $r \in(0,1)$. Then for any $f \in L^{p}(\mu)$, and $\varphi_{k}$ an approximate identity sequence $\left(\varphi_{k} \in C_{c}^{\infty}\left(\mathbb{R}^{n}\right)\right.$ with $\int \varphi_{k}(x) d x=1$ and $\left.\operatorname{supp} \varphi_{k} \downarrow\{0\}\right)$,

$$
\left\|f_{r} * \varphi_{k}-f_{r}\right\|_{L^{p}(\mu)} \rightarrow 0 \quad \text { as } \quad k \rightarrow \infty .
$$

Proof. Fix $\epsilon>0$ and let $\psi \in C_{c}\left(\mathbb{R}^{n}\right)$ be such that $\|f-\psi\|_{L^{p}(\mu)}<\epsilon$. The standard 3 term inequality in this case is

$$
\left\|f_{r} * \varphi_{k}-f_{r}\right\|_{L^{p}(\mu)} \leq\left\|\left(f_{r}-\psi_{r}\right) * \varphi_{k}\right\|_{L^{p}(\mu)}+\left\|\psi_{r} * \varphi_{k}-\psi_{r}\right\|_{L^{p}(\mu)}+\left\|\psi_{r}-f_{r}\right\|_{L^{p}(\mu)} .
$$

Following the proof of Lemma2.9, we have $\left\|f_{r}-\psi_{r}\right\|_{L^{p}(\mu)} \leq r^{-n / p} C_{\mu}(1 / r, 0)^{1 / p} \epsilon$, and from condition (1.2) of Definition 1.9 this is a uniformly bounded constant times $\epsilon$ for $r$ away from 0. Also, note that

$$
f_{r} * \varphi_{k}(x)=\int f_{r}(x-y) \varphi_{k}(y) d y=\int f(r x-r y) \varphi_{k}(y) d y=r^{-n} \int f(r x-u) \varphi_{k}(u / r) d u
$$

that is to say, $f_{r} * \varphi_{k}=r^{-n}\left(f * \tilde{\varphi}_{k}\right)_{r}$, where we set $\tilde{\varphi}_{k}=\left(\varphi_{k}\right)_{1 / r}$. Hence,

$$
\begin{aligned}
\left\|(f-\psi)_{r} * \tilde{\varphi}_{k}\right\|_{L^{p}(\mu)} & =r^{-n}\left\|\left((f-\psi) * \tilde{\varphi}_{k}\right)_{r}\right\|_{L^{p}(\mu)} \\
& \leq r^{-n} r^{-n / p} C_{\mu}\left(\frac{1}{r}, \frac{s_{k}}{r}\right)^{1 / p} \operatorname{Vol}\left(\operatorname{supp} \tilde{\varphi}_{k}\right)^{1 / p}\left\|\tilde{\varphi}_{k}\right\|_{L^{p^{\prime}\left(\mathbb{R}^{n}\right)}} \cdot\|f-\psi\|_{L^{p}(\mu)}
\end{aligned}
$$

by Proposition 2.7 where $s_{k}=\sup \left\{|w| ; w \in \operatorname{supp} \varphi_{k}\right\}$. Since $C_{\mu}\left(1 / r, \frac{s}{r}\right)$ is increasing in $s$, this constant is uniformly bounded as $k \rightarrow \infty$. What's more, cf. Remark 2.8 the product $\operatorname{Vol}\left(\operatorname{supp} \tilde{\varphi}_{k}\right)^{1 / p}\left\|\tilde{\varphi}_{k}\right\|_{L^{p^{\prime}}\left(\mathbb{R}^{n}\right)}$ can also be made constant with $k$ (for example by choosing $\varphi_{k}(x)=k^{n} \varphi(k x)$ for some fixed unit mass $C_{c}^{\infty}$ test-function $\varphi$ ). The result is that both the first and last terms in (2.6) are uniformly small as $k \rightarrow \infty$. Thus, we need only show that $\psi_{r} * \varphi_{k} \rightarrow \psi_{r}$ in $L^{p}(\mu)$. The quantity in question is the $p$ th root of

$$
\int\left|\int \psi_{r}(x-y) \varphi_{k}(y) d y-\psi_{r}(x)\right|^{p} \mu(d x)=\int\left|\int_{K_{k}}\left[\psi_{r}(x-y)-\psi_{r}(x)\right] \varphi_{k}(y) d y\right|^{p} \mu(d x),
$$

where we have used the fact that $\varphi_{k}$ is a probability density; here $K_{k}$ denotes the support of $\varphi_{k}$. Since $\psi_{r}$ is bounded, we may make the blunt estimate that the quantity in (2.7) is

$$
\leq \int \sup _{y \in K_{k}}\left|\psi_{r}(x-y)-\psi_{r}(x)\right|^{p}\left|\int_{K_{k}} \varphi_{k}(y) d y\right|^{p} \mu(d x)=\int \sup _{y \in K_{k}}\left|\psi_{r}(x-y)-\psi_{r}(x)\right|^{p} \mu(d x) .
$$

Since $\psi_{r}$ is continuous and $K_{k}$ is compact, there is a point $y_{k} \in K_{k}$ such that the supremum is achieved at $y_{k}$ : $\sup _{y \in K_{k}}\left|\psi_{r}(x-y)-\psi_{r}(x)\right|^{p}=\left|\psi_{r}\left(x-y_{k}\right)-\psi_{r}(x)\right|^{p}$. As $k \rightarrow \infty$, the support $K_{k}$ of $\psi_{k}$ shrinks to $\{0\}$, and so $y_{k} \rightarrow 0$. The function $\left|\psi_{r}\left(x-y_{k}\right)-\psi_{r}(x)\right|^{p}$ is continuous in $x$, and so converges to 0 pointwise as $y_{k} \rightarrow 0$. It therefore follows from the dominated convergence theorem that $\left\|\psi_{r} * \varphi_{k}-\psi_{r}\right\|_{L^{p}(\mu)} \rightarrow 0$, completing the proof.

We will now use Proposition 2.7 and Corollary 2.10 to prove our main approximation theorem: that $L_{E}^{p}(\mu)$ is dense in $L^{p}(\mu)$ through log-subharmonic functions. 


\subsection{The Proof of Theorem 1.12}

Proof of Theorem 1.12 The basic idea of the proof is as follows: approximate a function $f \in \operatorname{LSH} \cap L^{p}(\mu)$ by $(f * \varphi)_{r}$, and let $\varphi$ run through an approximate identity sequence and $r$ tend to 1 . We show that the dilated convolution $(f * \varphi)_{r}$ is in LSH $\cap L_{E}^{p}(\mu)$, and that these may be used to approximate $f$ in $L^{p}$-sense.

Part 1: $(f * \varphi)_{r}$ is in LSH $\cap L_{E}^{p}(\mu)$. Let $\varphi \in C_{c}^{\infty}\left(\mathbb{R}^{n}\right)$ be a non-negative test function. Lemma 1.19 shows that $f * \varphi$ is $C^{\infty}$ and LSH. It is elementary to verify that the cone $C^{\infty} \cap \mathrm{LSH}$ is invariant under dilations $g \mapsto g_{r}$; hence the dilated convolution $(f * \varphi)_{r}$ is $C^{\infty}$ and LSH. For fixed $r<1$, Proposition 2.7 shows that $(f * \varphi)_{r}$ is in $L^{p}(\mu)$, since $f \in L^{p}(\mu)$. We must now apply the differential operator $E$. Note that $(f * \varphi)_{r}$ is $C^{\infty}$, and so

$$
E\left[(f * \varphi)_{r}\right](x)=x \cdot \nabla\left[(f * \varphi)_{r}\right](x)=\int r x \cdot \nabla \varphi(r x-y) f(y) d y .
$$

Decomposing $r x=(r x-y)+y$, we break this up as two terms

$$
E\left[(f * \varphi)_{r}\right](x)=\int(r x-y) \cdot \nabla \varphi(r x-y) f(y) d y+\int y \cdot \nabla \varphi(r x-y) f(y) d y .
$$

The first term is just $(f * E \varphi)_{r}(x)$, and since $E \varphi$ is also $C_{c}^{\infty}\left(\mathbb{R}^{n}\right)$, Proposition 2.7 bounds the $L^{p}$-norm of this term by the $L^{p}$-norm of $f$. Hence, it suffices to show that the second term in 2.8) defines an $L^{p}(\mu)$-function of $x$. We now proceed analogously to the proof of Proposition 2.7. Changing variables $u=r x-y$ for fixed $x$ in the internal integral and then using Hölder's inequality,

$$
\begin{aligned}
& \int_{\mathbb{R}^{n}}\left|\int_{\mathbb{R}^{n}} y \cdot \nabla \varphi(r x-y) f(y) d y\right|^{p} \rho(x) d x \\
= & \int_{\mathbb{R}^{n}}\left|\int_{K}(r x-u) \cdot \nabla \varphi(u) f(r x-u) d u\right|^{p} \rho(x) d x \\
\leq & \int_{\mathbb{R}^{n}}\left(\int_{K}|r x-u|^{p}|f(r x-u)|^{p} d u\right)\left(\int_{K}|\nabla \varphi(u)|^{p^{\prime}} d y\right)^{p / p^{\prime}} \rho(x) d x,
\end{aligned}
$$

where $K=\operatorname{supp} \varphi$. Note that $\|\nabla \varphi\|_{p^{\prime}}<\infty$ is a constant independent of $f$. So we must consider the double integral, to which we apply Fubini's theorem,

$$
\int_{\mathbb{R}^{n}}\left(\int_{K}|r x-u|^{p}|f(r x-u)|^{p} d u\right) \rho(x) d x=\int_{K}\left(\int_{\mathbb{R}^{n}}|r x-u|^{p}|f(r x-u)|^{p} \rho(x) d x\right) d u .
$$

Now we change variables $v=r x-u$ for fixed $u$ in the internal integral, to achieve

$$
\int_{K}\left(\int_{\mathbb{R}^{n}}|v|^{p}|f(v)|^{p} \rho\left(\frac{v+u}{r}\right) r^{-n} d v\right) d u
$$

Finally, we utilize the assumption that $\rho$ is exponential type $p$, and so there is a constant $C(p, r, K)$ so that $|v|^{p} \rho\left(\frac{v+u}{r}\right) \leq C(p, r, K) \rho(u)$ for $u \in K$. Hence the integral in (2.9) is bounded above by $C(p, r, K) r^{-n} \operatorname{Vol}(K)$ times the finite norm $\int|f|^{p} d \mu$, which demonstrates that $E\left[(f * \varphi)_{r}\right]$ is in $L^{p}(\mu)$.

Part 2: $(f * \varphi)_{r}$ approximates $f$ in $L^{p}(\mu)$. Let $\varphi_{k}$ be an approximate identity sequence. Note by simple change of variables that $\left(f * \varphi_{k}\right)_{r}=f_{r} *\left(r^{n} \varphi_{k}\right)_{r}$, and that $\left(r^{n} \varphi_{k}\right)_{r}$ is also an approximate identity sequence. Since $f_{r} \in L^{p}(\mu)$, by Lemma 2.5 it follows from Corollary 2.10 that $\left(f * \varphi_{k}\right)_{r} \rightarrow f_{r}, k \rightarrow \infty$, in $L^{p}(\mu)$. We must now show that $f_{r} \rightarrow f$ in $L^{p}(\mu)$ as $r \uparrow 1$. For this purpose, once again fix $\epsilon>0$ and choose a $\psi \in C_{c}\left(\mathbb{R}^{n}\right)$ so that $\|f-\psi\|_{L^{p}(\mu)}<\epsilon$. Then

$$
\left\|f-f_{r}\right\|_{L^{p}(\mu)} \leq\|f-\psi\|_{L^{p}(\mu)}+\left\|\psi-\psi_{r}\right\|_{L^{p}(\mu)}+\left\|\psi_{r}-f_{r}\right\|_{L^{p}(\mu)} .
$$


The first term is $<\epsilon$, and changing variables the last term is

$$
\begin{aligned}
\left\|\psi_{r}-f_{r}\right\|_{L^{p}(\mu)}^{p}=\int|\psi(r x)-f(r x)|^{p} \rho(x) d x & =r^{-n} \int|\psi(u)-f(u)|^{p} \rho(u / r) d u \\
& \leq r^{-n} C_{\mu}\left(\frac{1}{r}, 0\right) \int|\psi-f|^{p} d \mu .
\end{aligned}
$$

Here we have used the fact that $\mu$ is Euclidean regular. Note that, by condition (1.2) of Definition 1.9 , the constant appearing here is uniformly bounded by, say, $C$, for $r \in\left(\frac{1}{2}, 1\right]$. Thence, the last term in (2.10) is bounded above by $C^{1 / p} \epsilon$ and is also uniformly small. Finally, the middle term tends to 0 as $r \uparrow 1$ since $\psi_{r} \rightarrow \psi$ pointwise and the integrand is uniformly bounded. Letting $\epsilon$ tend to 0 completes the proof.

\section{The Intrinsic Equivalence of (sLSI) and (sHC)}

In this section, we prove Theorem 1.14; if a measure $\mu$ is sufficiently Euclidean regular (satisfying the conditions of Definition 1.9), and if $\mu$ is invariant under rotations, then $\mu$ satisfies a strong log-Sobolev inequality precisely when it satisfies strong hypercontractivity. It will be useful to fix the following notation.

Notation 3.1. Let $c>0$ be a fixed constant, let $\mu$ be a measure on $\mathbb{R}^{n}$, and let $f$ be a function on $\mathbb{R}^{n}$.

1. For $r \in(0,1]$, let $q=q(r)$ denote the function

$$
q(r)=r^{-2 / c} .
$$

Note that $q \in C^{\infty}(0,1]$, is decreasing, and $q(1)=1$.

2. Define a function $\alpha_{f, \mu}:(0,1] \rightarrow[0, \infty)$ by

$$
\alpha_{f, \mu}(r) \equiv\left\|f_{r}\right\|_{L^{q(r)}(\mu)}=\left(\int|f(r x)|^{q(r)} \mu(d x)\right)^{1 / q(r)} .
$$

When the function $f$ and measure $\mu$ are clear from context, we denote $\alpha_{f, \mu}=\alpha$.

We begin with the following general statement.

Lemma 3.2. Suppose $\mu$ is a Euclidean regular probability measure. Let $q_{0}>1$, and let $f \geq 0$ be in $L^{q_{0}}(\mu) \cap$ $C^{\infty}\left(\mathbb{R}^{n}\right)$. Let $\epsilon \in(0,1)$, and suppose there are functions $h_{1}, h_{2} \in L^{1}(\mu)$ such that for all $r \in(\epsilon, 1]$,

$$
\left|f(r x)^{q(r)} \log f(r x)\right| \leq h_{1}(x), \quad\left|f(r x)^{q(r)-1} E f(r x)\right| \leq h_{2}(x) \quad \text { a.s. }[x] .
$$

Then there is $\epsilon^{\prime} \in(\epsilon, 1)$ such that $\alpha=\alpha_{f, \mu}$ is differentiable on $\left(\epsilon^{\prime}, 1\right]$, and for $r$ in this domain,

$$
\begin{aligned}
\alpha^{\prime}(r)=\frac{2}{c r q(r)}\left\|f_{r}\right\|_{q(r)}^{1-q(r)}\left[\left\|f_{r}\right\|_{q(r)}^{q(r)} \log \left\|f_{r}\right\|_{q(r)}^{q(r)}\right. & -\int f(r x)^{q(r)} \log f(r x)^{q(r)} \mu(d x) \\
& \left.+\frac{c q(r)}{2} \int f(r x)^{q(r)-1} \operatorname{Ef}(r x) \mu(d x)\right] .
\end{aligned}
$$

Remark 3.3. Note that $(1 / q(r))^{c / 2}=r$. Hence, if $f \in \mathrm{LSH}$ and $\mu$ satisfies the strong hypercontractivity property of (SHC) (with $p=1$ ) we have $\alpha(r) \leq\|f\|_{1}=\alpha(1)$ for $r \in(0,1]$. The conditions of Lemma 3.2 guarantee that $\alpha$ is differentiable; hence, we essentially have that $\alpha^{\prime}(1) \geq 0$. Equation (3.2) shows that $\alpha^{\prime}(1)$ is closely related to the expression in (SLSI), and indeed this is our method for proving the logarithmic Sobolev inequality in what follows. 
Proof. Set $\beta(r, x)=f(r x)^{q(r)}$, so that $\alpha(r)^{q(r)}=\int \beta(r, x) \mu(d x)$. Note, $\beta(r, x)=f_{r}(x)^{q(r)}$. The function $q(r)$ is continuous and $q(1)=1$, so there is $\epsilon^{\prime}>0$ so that $q(r)<q_{0}$ for $r \in\left(\epsilon^{\prime}, 1\right)$; and hence $f^{q(r)} \in L^{1}(\mu)$. (We increase $\epsilon^{\prime}$ if necessary so $0<\epsilon<\epsilon^{\prime}$.) As $\mu$ is Euclidean regular, Lemma 2.5 shows that $f_{r}^{q(r)}$ is also in $L^{1}(\mu)$, and so $\beta(r, \cdot) \in L^{1}(\mu)$ for all $r \in\left(\epsilon^{\prime}, 1\right)$. Since $f \in C^{\infty}$, we can check quickly that $\beta(\cdot, x)$ is as well; using the fact that $q^{\prime}(r)=-\frac{2}{c} r^{-2 / c-1}=-\frac{2}{c r} q(r)$, and that $\frac{\partial}{\partial r} f(r x)=\frac{1}{r} E f(r x)$, logarithmic differentiation yields

$$
\frac{\partial}{\partial r} \beta(r, x)=q(r)\left[-\frac{2}{c r} f(r x)^{q(r)} \log f(r x)+\frac{1}{r} f(r x)^{q(r)-1} E f(r x)\right] .
$$

From the hypotheses of the Lemma, we therefore have

$$
\left|\frac{\partial}{\partial r} \beta(r, x)\right| \leq \frac{q(r)}{r}\left[\frac{2}{c} h_{1}(x)+h_{2}(x)\right]
$$

for almost every $x \in \mathbb{R}^{n}$, for $r \in\left(\epsilon^{\prime}, 1\right]$. As $q(r) / r$ is uniformly bounded on $\left(\epsilon^{\prime}, 1\right]$, we see that $\left|\frac{\partial}{\partial r} \beta(r, x)\right|$ is uniformly bounded above by an $L^{1}(\mu)$ function. It now follows from the Lebesgue differentiation theorem that $\alpha(r)^{q(r)}=\int \beta(r, x) \mu(d x)$ is differentiable on a neighbourhood of 1 , and

$$
\begin{aligned}
\frac{d}{d r}\left[\alpha(r)^{q(r)}\right] & =\int \frac{\partial}{\partial r} \beta(r, x) \mu(d x) \\
& =-\frac{2}{c r} q(r) \int f(r x)^{q(r)} \log f(r x) \mu(d x)+\frac{1}{r} q(r) \int f(r x)^{q(r)-1} \operatorname{Ef}(r x) \mu(d x) .
\end{aligned}
$$

Consequently $\alpha(r)$ is differentiable in a neighbourhood of 1 . Again using logarithmic differentiation,

$$
\alpha^{\prime}(r)=\alpha(r) \frac{d}{d r} \log \alpha(r)=\alpha(r) \frac{d}{d r}\left[\frac{1}{q(r)} \log \alpha(r)^{q(r)}\right]
$$

and again using the fact that $q^{\prime}(r)=-\frac{2}{c r} q(r)$,

$$
\begin{aligned}
\frac{d}{d r}\left[\frac{1}{q(r)} \log \alpha(r)^{q(r)}\right] & =\frac{2}{c r q(r)} \log \alpha(r)^{q(r)}+\frac{1}{q(r)} \alpha(r)^{-q(r)} \frac{d}{d r}\left[\alpha(r)^{q(r)}\right] \\
& =\frac{\alpha(r)^{-q(r)}}{q(r)}\left(\frac{2}{c r} \alpha(r)^{q(r)} \log \alpha(r)^{q(r)}+\frac{d}{d r}\left[\alpha(r)^{q(r)}\right]\right) .
\end{aligned}
$$

Combining with (3.4), we therefore have

$$
\begin{aligned}
\alpha^{\prime}(r)=\frac{\alpha(r)^{1-q(r)}}{q(r)}\left[\frac{2}{c r} \alpha(r)^{q(r)} \log \alpha(r)^{q(r)}\right. & -\frac{2}{c r} q(r) \int f(r x)^{q(r)} \log f(r x) \mu(d x) \\
& \left.+\frac{1}{r} q(r) \int f(r x)^{q(r)-1} \operatorname{Ef}(r x) \mu(d x)\right]
\end{aligned}
$$

Simplifying (3.5), and using the definition $\alpha(r)=\left\|f_{r}\right\|_{q(r)}$, yields (3.2), proving the lemma.

We therefore seek conditions on a function $f$ (and on the measure $\mu$ ) which guarantee the hypotheses of Lemma 3.2 (specifically the existence of the Lebesgue dominating functions $h_{1}$ and $h_{2}$ ). Naturally, we will work with LSH functions $f$. We will also make the fairly strong assumption that $\mu$ is rotationally-invariant.

Notation 3.4. Let $f: \mathbb{R}^{n} \rightarrow \mathbb{R}$ be locally-bounded. Denote by $\tilde{f}$ the spherical average of $f$. That is, with $\vartheta$ denoting Haar measure on the group $O(n)$ of rotations of $\mathbb{R}^{n}$,

$$
\tilde{f}(x)=\int_{O(n)} f(u x) \vartheta(d u) .
$$


If $\mu$ is rotationally-invariant, then $\int f d \mu=\int \tilde{f} d \mu$ for any $f \in L^{1}(\mu)$. As such, we can immediately weaken the integrability conditions of Lemma 3.2 as follows.

Lemma 3.5. Suppose $\mu$ is a Euclidean regular probability measure that is invariant under rotations of $\mathbb{R}^{n}$. Let $q_{0}>1$ and let $f \geq 0$ be in $L^{q_{0}}(\mu) \cap C^{\infty}\left(\mathbb{R}^{n}\right)$. Denote by $f_{1}, f_{2}:(0,1] \times \mathbb{R}^{n} \rightarrow \mathbb{R}$ the functions

$$
f_{1}(r, x)=f(r x)^{q(r)} \log f(r x), \quad f_{2}(r, x)=f(r x)^{q(r)-1} E f(r x) .
$$

Fix $\epsilon \in(0,1)$, and suppose that there exist functions $h_{1}, h_{2} \in L^{1}(\mu)$ such that, for $r \in(\epsilon, 1],\left|\tilde{f}_{j}(r, x)\right| \leq h_{j}(x)$ for almost every $x, j=1,2$. (Here $\tilde{f}_{j}(r, \cdot)$ refers to the rotational average of $f_{j}(r, \cdot)$, as per Notation 3.4 ) Then the conclusion of Lemma 3.2 stands: for some $\epsilon^{\prime} \in(\epsilon, 1)$, the function $\alpha=\alpha_{f, \mu}$ is differentiable on $\left(\epsilon^{\prime}, 1\right]$, and its derivative is given by (3.2).

Proof. Following the proof of Lemma 3.2, only a few modifications are required. Defining $\beta(r, x)$ as above, $\alpha(r)^{q(r)}=\int \beta(r, x) \mu(d x)$; since $\mu$ is rotationally-invariant, this is equal to $\int \tilde{\beta}(r, x) \mu(d x)$ where $\tilde{\beta}$ refers to the rotational average of $\beta$ in the variable $x$. Evidently $\tilde{\beta}(r, \cdot)$ is $\mu$-integrable for sufficiently large $r<1$ (since $\beta$ is). To use the Lebesgue differentiation technique, we must verify that $\frac{\partial}{\partial r} \tilde{\beta}(r, x)$ exists for almost every $x$ and is uniformly bounded by an $L^{1}(\mu)$ dominator. Note that $\beta(r, x)$ is locally-bounded in $x$ for each $r$, and so for fixed $x$ it is easy to verify that indeed

$$
\frac{\partial}{\partial r} \tilde{\beta}(r, x)=\int_{O(n)} \frac{\partial}{\partial r} \beta(r, u x) \vartheta(d u) .
$$

Using (3.3), we then have

$$
\frac{\partial}{\partial r} \tilde{\beta}(r, x)=q(r) \int_{O(n)}\left(-\frac{2}{c r} f(r u x)^{q(r)} \log f(\text { rux })+\frac{1}{r} f(r u x)^{q(r)-1} \operatorname{Ef}(\text { rux })\right) \vartheta(d u) .
$$

That is, using (3.6), $\frac{\partial}{\partial r} \tilde{\beta}(r, x)=q(r)\left[-\frac{2}{c r} \tilde{f}_{1}(r, x)+\frac{1}{r} \tilde{f}_{2}(r, x)\right]$. Hence, from the assumptions of this lemma,

$$
\left|\frac{\partial}{\partial r} \tilde{\beta}(r, x)\right| \leq \frac{q(r)}{r}\left[\frac{2}{c} h_{1}(x)+h_{2}(x)\right]
$$

and so, since $q(r) / r$ is uniformly bounded for $r \in\left(\frac{1}{2}, 1\right]$, it follows that $\alpha(r)^{q(r)}=\int \tilde{\beta}(r, x) \mu(d x)$ is differentiable near 1 , with derivative given by

$$
\int \frac{\partial}{\partial r} \tilde{\beta}(r, x) \mu(d x)=q(r)\left[-\frac{2}{r c} \int \tilde{f}_{1}(r, x) \mu(d x)+\frac{1}{r} \int \tilde{f}_{2}(r, x) \mu(d x) .\right]
$$

Now using the rotational-invariance of $\mu$ again, these integrals are the same as the corresponding non-rotated integrands $\int f_{j}(r, x) \mu(d x)$, yielding the same result as (3.4). The remainder of the proof follows the proof of Lemma 3.2 identically.

Remark 3.6. The point of Lemma3.5- that it is sufficient to find uniform Lebesgue dominators for the rotational averages of the terms in (3.1) - is actually quite powerful for us. While a generic subharmonic function in dimension $\geq 2$ may not have good global properties, a rotationally-invariant subharmonic function does, as the next proposition demonstrates. We will exploit this kind of behaviour to produce the necessary bounds to verify the conditions of Lemma 3.5 and prove the differentiability of the norm.

Proposition 3.7. Let $f: \mathbb{R}^{n} \rightarrow \mathbb{R}$ be subharmonic and locally-bounded. Then $\tilde{f}$ is also subharmonic; moreover, for fixed $x \in \mathbb{R}^{n}, r \mapsto \tilde{f}(r x)$ is an increasing function of $r \in[0,1]$. 
Proof. Fix $u \in O(n)$. Since $f$ is locally-bounded, subharmonicity means that $f_{B(x, r)} f(t) d t \geq f(x)$ for every $x \in \mathbb{R}^{n}, r \in(0, \infty)$. Changing variables, we have

$$
f_{B(x, r)} f(\alpha t) d t=f_{u \cdot B(x, r)} f(t) d t=f_{B(u x, r)} f(t) d t \geq f(u x) .
$$

Hence, $f \circ u$ is subharmonic for each $u \in O(n)$. The local-boundedness of $f$ means that the function $u \mapsto f(u x)$ is uniformly bounded in $L^{1}(O(n), \vartheta)$ for $x$ in a compact set, and hence it follows that $\tilde{f}$ is subharmonic.

Hence $\tilde{f}$ is a rotationally-invariant subharmonic function. Fix $x \in \mathbb{R}^{n}$ and $r \in[0,1]$. Then $r x$ is in the ball $B(0,|x|)$, and since $\tilde{f}$ is subharmonic, the maximum principle asserts that $\tilde{f}(r x)$ is no larger than the maximum of $f$ on $\partial B(0,|x|)$. But $\tilde{f}$ is constantly equal to $\tilde{f}(x)$ on $\partial B(0,|x|)$ by rotational-invariance, and so $\tilde{f}(r x) \leq \tilde{f}(x)$, proving the proposition.

Proposition 3.7 makes it quite easy to provide a uniform Lebesgue dominating function for the function $f_{1}$ in Lemma 3.5 .

Proposition 3.8. Suppose $\mu$ is a rotationally-invariant probability measure on $\mathbb{R}^{n}$. Let $q_{0}>1$, and let $f \geq 0$ be subharmonic and in $L^{q_{0}}(\mu)$. Define $f_{1}$ as in (3.6): $f_{1}(r, x)=f(r x)^{q(r)} \log f(r x)$. Set $g_{1}(x)=f(x)^{q_{0}}$, and set $h_{1}=\tilde{g_{1}}+1$; i.e. $h_{1}(x)=1+\int_{O(n)} f(u x)^{q_{0}} \vartheta(d u)$. Then $h_{1} \in L^{1}(\mu)$ and there is an $\epsilon \in(0,1)$ and a constant $C>0$ so that for all $r \in(\epsilon, 1],\left|\tilde{f}_{1}(r, x)\right| \leq C h_{1}(x)$ for almost every $x$.

Remark 3.9. By the rotational-invariance of $\mu, \int h_{1} d \mu=\int \tilde{g_{1}} d \mu+1=\int g_{1} d \mu+1=\int f^{q_{0}} d \mu+1<\infty$, and so $h_{1}$ is a uniform $L^{1}(\mu)$ dominator verifying the first condition of Lemma 3.5 .

Proof. Choose some small $\delta \in(0,1)$. First note from simple calculus that, for $u \geq 1, u^{-\delta} \log u \leq \frac{1}{e \delta}$. Now, choose $\epsilon \in(0,1)$ so that $q \epsilon<q_{0}-\delta$; then $q(r)<q_{0}-\delta$ for $r \in(\epsilon, 1]$. Consequently, if $f(y) \geq 1$, we have

$$
0 \leq f(y)^{q(r)} \log f(y) \leq f(y)^{q_{0}-\delta} \log f(y) \leq \frac{1}{e \delta} f(y)^{q_{0}} .
$$

On the other hand, for $0 \leq u \leq 1,\left|u^{q(r)} \log u\right| \leq \frac{1}{e q(r)} \leq \frac{1}{e}$ (again by simple calculus). Thus, since $f \geq 0$, in total we have

$$
\left|f(y)^{q(r)} \log f(y)\right| \leq \frac{1}{e} \max \left\{\frac{1}{\delta} f(y)^{q_{0}}, 1\right\} \leq \frac{1}{e \delta}\left[f(y)^{q_{0}}+1\right] .
$$

Set $C=\frac{1}{e \delta}$. With $y=r x$, the left-hand-side of (3.7) is precisely $f_{1}(r, x)$. Averaging (3.7) over $O(n)$ and recalling that $g_{1}(y)=f(y)^{q_{0}}$, we have

$$
\mid \tilde{f}_{1}(r, x) \leq C\left[\tilde{g_{1}}(r x)+1\right]
$$

Recall that if $\varphi$ is convex and $f$ is subharmonic then $\varphi \circ f$ is also subharmonic. Thus, since $q_{0}>1$ and $f$ is subharmonic, $g_{1}$ is also subharmonic, and hence from Proposition $3.7 \tilde{g_{1}}(r x) \leq \tilde{g_{1}}(x)$. This proves the proposition.

We must now bound the second term $\tilde{f}_{2}(r, \cdot)$ uniformly for $r$ in a neighbourhood of 1 . The following Lemma is useful in this regard.

Lemma 3.10. Let $\tilde{k}$ be a $C^{\infty}$ non-negative subharmonic rotationally-invariant function. Then for $x \in \mathbb{R}^{n}$ and $r \in(0,1]$,

$$
E \tilde{k}(r x) \leq r^{2-n} E \tilde{k}(x)
$$


Proof. Since $\tilde{k}$ is rotationally-invariant, there is a function $h:[0, \infty) \rightarrow \mathbb{R}$ so that $\tilde{k}(x)=h(|x|)$. The Laplacian of $\tilde{k}$ can then be expressed in terms of derivatives of $h$; the result is

$$
\Delta \tilde{k}(x)=h^{\prime \prime}(|x|)+(n-1) \frac{1}{|x|} h^{\prime}(|x|) .
$$

Hence, since $\tilde{k}$ is subharmonic and smooth, it follows that for $t>0$,

$$
t h^{\prime \prime}(t)+(n-1) h^{\prime}(t) \geq 0 .
$$

One can also check that, in this case, $E \tilde{k}(x)=|x| h^{\prime}(|x|)$. Now, define $F(r)=r^{n-2} E \tilde{k}(r x)=r^{n-2} r|x| h^{\prime}(r|x|)$. Then $F$ is smooth on $(0, \infty)$ and $F(1)=|x| h^{\prime}(|x|)=E \tilde{k}(x)$. We differentiate, yielding

$$
\begin{aligned}
F^{\prime}(r) & =|x| \frac{d}{d r} r^{n-1} h^{\prime}(r|x|)=|x|(n-1) r^{n-2} h^{\prime}(r|x|)+|x| r^{n-1} h^{\prime \prime}(r|x|)|x| \\
& =|x| r^{n-2}\left[r|x| h^{\prime \prime}(r|x|)+(n-1) h^{\prime}(r|x|)\right] .
\end{aligned}
$$

Equation (3.10) with $t=r|x|$ now yields that $F^{\prime}(r) \geq 0$ for $r>0$. Hence, $F(r) \leq F(1)$ for $r \leq 1$. This is precisely the statement of the lemma.

Proposition 3.11. Let $q_{0}>1$ and let $\mu$ be a rotationally-invariant probability measure on $\mathbb{R}^{n}$. Let $f \geq 0$ be subharmonic, $C^{\infty}$, and in $L_{E}^{q_{0}}(\mu)$. Define $f_{2}$ as in (3.6): $f_{2}(r, x)=f(r x)^{q(r)-1} E f(r x)$. Set $g_{3}(x)=$ $\left(f(x)^{q_{0}-1}+1\right)|E f(x)|$, and set $h_{2}=\tilde{g_{3}}$. Then there is an $\epsilon \in(0,1)$ and a constant $C>0$ so that for all $r \in(\epsilon, 1],\left|\tilde{f}_{2}(r, x)\right| \leq C h_{2}(x)$ for almost every $x$; moreover, $h_{2} \in L^{1}(\mu)$.

Proof. Fix $\epsilon \in(0,1)$ small enough that $q(r)<q_{0}$ for all $r \in(\epsilon, 1]$. Define $g_{2}(r, y)=f(y)^{q(r)-1} E f(y)$. and note that $f_{2}(r, x)$ is given by the dilation $f_{2}(r, x)=g_{2}(r, r x)$. Since $E$ is a first-order differential operator, we can quickly check that

$$
g_{2}(r, y)=\frac{1}{q(r)} E\left(f^{q(r)}\right)(y)
$$

We now average both sides over $O(n)$. Set $k=f^{q(r)}$, which is $C^{\infty}$, and let $u \in O(n)$. Then we have the following calculus identity:

$$
E(k \circ u)(y)=y \cdot \nabla(k \circ u)(y)=y \cdot u^{\top} \nabla k(u y)=(u y) \cdot \nabla k(u y)=(E k)(u y) .
$$

For fixed $y$ the function $u \mapsto(E k)(u y)$ is uniformly bounded and so we integrate both sides to yield

$$
\widetilde{E k}(y)=\int_{O(n)}(E k)(u y) \vartheta(d u)=\int E(k \circ u)(y) \vartheta(d u)=E \int k \circ u(y) \vartheta(d u)=E(\tilde{k})(y) .
$$

In other words, $\tilde{g_{2}}(r, y)=\frac{1}{q(r)} E\left(\widetilde{f^{q(r)}}\right)(y)$. As in the proof of Proposition 3.8 the function $\tilde{k}=\widetilde{f^{q(r)}}$ is subharmonic, and rotationally invariant. Hence, we employ Lemma 3.10 and have

$$
\tilde{g_{2}}(r, r x)=\frac{1}{q(r)} E \tilde{k}(r x) \leq \frac{1}{q(r)} r^{2-n} E \tilde{k}(x)=r^{2-n} \tilde{g_{2}}(r, x) .
$$

Since $r^{2-n}$ is uniformly bounded for $r \in(\epsilon, 1]$, it now suffices to find a uniform dominator for $\tilde{g_{2}}(r, x)$.

We therefore make the estimates: since $q(r)<q_{0}$ we have

$$
\begin{aligned}
\left|g_{2}(r, x)\right|=f(x)^{q(r)-1}|E f(x)| \leq \max \left\{f(x)^{q(r)-1}, 1\right\}|E f(x)| & \leq \max \left\{f(x)^{q_{0}-1}, 1\right\}|E f(x)| \\
& \leq\left(f(x)^{q_{0}-1}+1\right)|E f(x)| .
\end{aligned}
$$


That is to say, $\left|g_{2}(r, x)\right| \leq g_{3}(x)$ for $r \in(\epsilon, 1]$. Hence,

$$
\left|\tilde{g_{2}}(r, x)\right|=\left|\int_{O(n)} g_{2}(r, u x) \vartheta(d u)\right| \leq \int_{O(n)}\left|g_{2}(r, u x)\right| \vartheta(d u) \leq \int_{O(n)} g_{3}(u x) \vartheta(d u)=\tilde{g_{3}}(x)=h_{2}(x),
$$

thus proving the estimate.

As usual, by rotational invariance of $\mu, \int \tilde{g_{3}} d \mu=\int g_{3} d \mu$, and so to show $h_{2} \in L^{1}(\mu)$ we need only verify that $g_{3} \in L^{1}(\mu)$. To that end, we break up $g_{3}(x)=f(x)^{q_{0}-1}|E f(x)|+|E f(x)|$. By assumption, $f \in L_{E}^{q_{0}}(\mu)$ and so $|E f| \in L^{q_{0}}(\mu)$; as $\mu$ is a finite measure, this means that $|E f| \in L^{1}(\mu)$ and hence the second term is integrable.

For the first term, we use Hölder's inequality:

$$
\int f^{q_{0}-1}|E f| d \mu \leq\left\|f^{q_{0}-1}\right\|_{q_{0}^{\prime}}\|E f\|_{q_{0}}=\|f\|_{q_{0}}^{q_{0}-1}\|E f\|_{q_{0}} .
$$

Both terms are finite since $f \in L_{E}^{q_{0}}(\mu)$, and hence $g_{3} \in L^{1}(\mu)$, proving the proposition.

Combining Lemma 3.5 and Propositions 3.8 and 3.11, we therefore have the following.

Theorem 3.12. Let $q_{0}>1$ and let $\mu$ be a probability measure of Euclidean type $q_{0}$, that is invariant under rotations of $\mathbb{R}^{n}$. Suppose that $\mu$ satisfies strong hypercontractivity of $(\underline{S H C})$ with constant $c>0$. Let $f \in$ $L_{E}^{q_{0}}(\mu) \cap \mathrm{LSH} \cap C^{\infty}$. Then the strong log-Sobolev inequality, ([SLSI), holds for $f$ :

$$
\int f \log f d \mu-\int f d \mu \log \int f d \mu \leq \frac{c}{2} \int E f d \mu .
$$

Proof. Under the conditions stated above, the results of the preceding section show that the function $\alpha=\alpha_{f, \mu}$ is differentiable on $\left(\epsilon^{\prime}, 1\right]$ for some $\epsilon^{\prime} \in(0,1)$. Since $\mu$ satisfies strong hypercontractivity, Proposition 1.16 shows that the function $\alpha$ is non-decreasing on $(0,1]$. It therefore follows that $\alpha^{\prime}(r) \geq 0$ for $r \in\left(\epsilon^{\prime}, 1\right]$ (here $\alpha^{\prime}(1)$ denotes the left-derivative). Hence, from (3.2) we have, for $r \in\left(\epsilon^{\prime}, 1\right]$,

$$
\left\|f_{r}\right\|_{q(r)}^{q(r)} \log \left\|f_{r}\right\|_{q(r)}^{q(r)}-\int f(r x)^{q(r)} \log f(r x)^{q(r)} \mu(d x)+\frac{c q(r)}{2} \int f(r x)^{q(r)-1} E f(r x) \mu(d x) \geq 0 .
$$

At $r=1$, this reduces precisely to (SLSI), proving the result.

Theorem 3.12 is part (2) of Theorem 1.14 The proof of (1) is essentially the same.

\section{References}

[1] Anderson, G.; Guionnet, A.; Zeitouni, O.: An introduction to random matrices. Cambridge Studies in Advanced Mathematics, 118. Cambridge University Press, Cambridge, 2010.

[2] Bakry, D.: L'hypercontractivité et son utilisation en théorie des semigroupes. Lectures on probability theory (Saint-Flour, 1992), 1-114, Lecture Notes in Math., 1581, Springer, Berlin, 1994.

[3] Bakry, D.: On Sobolev and logarithmic Sobolev inequalities true for Markov semigroups. New trends in stochastic analysis (Charingworth, 1994), 43-75, World Sci. Publ., River Edge, NJ, 1997.

[4] Bakry, D.; Emery, M.: Diffusions hypercontractives. Séminaire de Probabilité XIX. Lecture Notes in Math. 1123, 179-206 (1985) 
[5] Bobkov, S.; Houdré, Christian: Some connections between isoperimetric and Sobolev-type inequalities. Mem. Amer. Math. Soc. 129 no. 616 (1997)

[6] Bobkov, S.; Ledoux, M.: From Brunn-Minkowski to Brascamp-Lieb and to logarithmic Sobolev inequalities. Geom. Funct. Anal. 10, no. 5, 1028-1052 (2000)

[7] Bobkov, S.; Tetali, P.: Modified logarithmic Sobolev inequalities in discrete settings. J. Theoret. Probab. 19, no. 2, 289-336 (2006)

[8] Davies, E. B.: Explicit constants for Gaussian upper bounds on heat kernels. Amer. J. Math. 109, no. 2, 319-333 (1987)

[9] Davies, E. B.: Heat kernels and spectral theory. Cambridge Tracts in Mathematics, 92. Cambridge University Press, 1990.

[10] Davies, E. B., Simon, B.: Ultracontractivity and the heat kernel for Schrödinger operators and Dirichlet Laplacians. J. Funct. Anal. 59, 335-395 (1984)

[11] Diaconis, P., Saloff-Coste, L.: Logarithmic Sobolev inequalities for finite Markov chains. Ann. Appl. Probab. 6, 695-750 (1996)

[12] Graczyk, P.; Kemp, T.; Loeb, J.J.: Hypercontractivity for log-subharmonic functions. J. Funct. Anal. 258 (2010), 1785-1805.

[13] Galaz-Fontes, F.; Gross, L.; Sontz, S.: Reverse hypercontractivity over manifolds. Ark. Math., 39 2001, 283-309.

[14] Gross, L.: Logarithmic Sobolev inequalities. Amer. J. Math. 97 1975, 1061-1083.

[15] Gross, L.: Hypercontractivity over complex manifolds, Acta Mathematica, 182, 2000, 159-206.

[16] Gross, L.; Grothaus, M.: Reverse hypercontractivity for subharmonic functions. Canad. J. Math. 57 2005, 506-534.

[17] Guionnet, A.; Zegarlinski, B.: Lectures on logarithmic Sobolev inequalities. Séminaire de Probabilités, XXXVI, 1-134, Lecture Notes in Math., 1801, Springer, Berlin, 2003.

[18] Holley, R., Stroock, D.: Logarithmic Sobolev inequalities and stochastic Ising models. J. Statist. Phys. 46, 1159-1194 (1987)

[19] Janson, S.: On hypercontractivity for multipliers of orthogonal polynomials. Ark. Mat, 21 1983, 97-110.

[20] Janson, S.: On complex hypercontractivity. J. Funct. Anal., 151 1997, 270-280.

[21] Ledoux, M.: Isoperimetry and Gaussian analysis. Lectures on probability theory and statistics. 165-294, Lecture Notes in Math 1648, Springer, Berlin, 1996

[22] Ledoux, M.: Concentration of measure and logarithmic Sobolev inequalities. Séminaire de Probabilités, XXXIII, 120-216, Lecture Notes in Math., 1709, Springer, Berlin, 1999.

[23] Ledoux, M.: The geometry of Markov diffusion generators. Ann. Fac. Sci. Toulouse Math. (6) 9, no. 2 , 305-366 (2000)

[24] Ledoux, M.: The concentration of measure phenomenon. Mathematical Surveys and Monographs, 89. American Mathematical Society, Providence, RI, 2001. 
[25] Ledoux, M.: A remark on hypercontractivity and tail inequalities for the largest eigenvalues of random matrices. Séminaire de Probabilités XXXVII, 360369, Lecture Notes in Math., 1832, Springer, Berlin, 2003.

[26] Ledoux, M.: Spectral gap, logarithmic Sobolev constant, and geometric bounds. Surveys in differential geometry. Vol. IX, 219240, Surv. Differ. Geom., IX, Int. Press, Somerville, MA, 2004.

[27] Lelong, P.; Gruman, L.: Entire Functions of Several Complex Variables. Grundlehren der Mathematischen Wissenschaften [Fundamental Principles of Mathematical Sciences], 282. Springer-Verlag, Berlin, 1986.

[28] Nelson, E.: The free Markov field. J. Funct. Anal., 12 1973, 211-227.

[29] Stam, A.: Some inequalities satisfied by the quantities of information of Fisher and Shannon. Information and Control 2, 101-112 (1959)

[30] Villani, C.: Topics in optimal transportation. Graduate Studies in Mathematics, 58. American Mathematical Society, Providence, RI, 2003.

[31] Yau, H.T.: Logarithmic Sobolev inequality for the lattice gases with mixing conditions. Commun. Math. Phys. 181, 367-408 (1996)

[32] Yau, H.T.: Log-Sobolev inequality for generalized simple exlcusion processes. Probab. Theory Related Fields 109, 507-538 (1997)

[33] Zegarlinski, B.: Dobrushin uniqueness theorem and logarithmic Sobolev inequalities. J. Funct. Anal. 105, 77-111 (1992)

[34] Zimmermann, D.: Logarithmic Sobolev inequalities for mollified compactly supported measures. J. Funct. Anal. 265 no. 6, 1064-1083 (2013) 